\title{
LPV Control for a Semi-Active Suspension Quarter of Car-One Parameter Case
}

\author{
Jorge de Jesús Lozoya Santos ${ }^{1}$, Juan Carlos Tudon-Martinez ${ }^{1}$ and Ricardo A. Ramirez-Mendoza ${ }^{2}$ \\ ${ }^{1}$ Dirección de Investigación, DIECI, Universidad de Monterrey, 66238 San Pedro Garza Garcia, Nuevo Leon, Mexico \\ ${ }^{2}$ Dirección de Investigación, Escuela de Ingeniería, Tecnológico de Monterrey 64810 Monterrey, Nuevo Leon, Mexico
}

\begin{abstract}
The actual semi-active suspension control systems with a balance between comfort and road holding goals are not optimal because in these solutions one goal or the other always dominates in the suspension performance. This paper is centered in a new proposal to control an automotive semi-active suspension to achieve the comfort and maintain the road holding. The output in the control strategy is the electric current. A nonlinear quarter of vehicle model simulation compares and validates the proposal versus different controllers. The controller is designed with the $\mathrm{H} \infty$ criteria and the Linear Varying Parameter (LPV) considering the saturation and sigmoid shape of the F-V characteristic diagram. Unlike the solutions in literature, which use at least two scheduling parameters, the proposed LPV controller scheme for a semi-active suspension uses only one scheduling parameter.
\end{abstract}

\section{Introduction}

A control strategy for semi-active suspension in a Quarter of Vehicle $(Q \circ V)$ consists in giving the control goals, and in developing the controller, and the algorithm to map the controller output to the electric current. Most of the semiactive suspension controllers are devoted to a specific goal, while in control theory the controllers are independent of the goals. Hence, in such cases, the control strategy consists in the selection of the controller and the mapping algorithm. Seminal results show that a semi-active suspension can decrease up to $52 \%$ of vertical sprung mass acceleration and up to $20 \%$ of vertical sprung mass displacement, [1].

Two general classifications of semi-active control exist. The first type is the Continuously variable SemiActive (CSA) control. The type of manipulation generates a continuous manipulation over an interval in the semiactive interface [2]. The second type is the On-off SemiActive (OSA) control. The manipulation has only two values in the semi-active interface, according to the applied damping coefficient: hard for road holding or soft for ride comfort, [3].

The controllers can be categorized according to the number of goals to optimize: comfort, road holding and deflection. Some controllers optimize one objective, while the actual goal is the compromise between comfort and road holding. Some recent works are focused on the decrease in the compromise between comfort and road holding [4]. An interesting strategy is the $\mathrm{LPV} / \mathrm{H} \infty$ approach which solves the compromise adequately and with low on-line computation, [1], [5]. In this paper, a controller for an automotive semi-active suspension adaptable to the controllable shock absorber nonlinearities in order to ensure the full exploiting of the damping variability will be developed. The design will specify the same units in the controller output and the manipulation input of the controllable damper and it will seek to reach the damping steady state to fully explore the semi-activeness. The proposed controller must ensure good performances in both comfort and road holding. The pseudo-Bode and transient response plots will be the qualitative criteria in the frequency and time domain respectively. The quantitative criteria are the Power Spectral Density (PSD) and the RMS.

\section{Controller design}

The actual semi-active suspension control systems with a balance between comfort and road holding goals are not optimal because in these solutions one goal or the other always dominates in the suspension performance. The control strategy considers the piston velocity and displacement as the control system inputs. These inputs ensure the mapping of nonlinear characteristics of the suspension in control laws. The output in the control strategy, in both controllers, is the electric current. A nonlinear $Q \circ V$ model simulation compares and validates the proposal versus different controllers.

The control system consists in a gain-scheduling approach. A simple $M R$ damper model is used allowing the scheduling of the damping coefficient nonlinearity in the control law. It is inspired in the work of [1] and [6]. The new contribution is the inclusion of a simple $M R$ damper model and the use of only one scheduling 
parameter. The controller is designed with the $\mathrm{H}_{\infty}$ criteria and the Linear Varying Parameter ( $L P V)$ considering the saturation and sigmoid shape of the $F-V$ characteristic diagram.

The electric current is the input signal that allows exploring in a full way the characteristics of the $M R$ damper. Using this signal as the controller output and the chosen inputs, a complete exploration of the semi-active zone in the damper characteristic is ensured. However, the use of the electric current as a controller output is not common. Most of the actual research works use the force or damping coefficient, and then a conversion algorithm for the electric current. This can be one of the causes of the actual compromise between comfort and road holding in semi-active suspensions.

\subsection{LPV/H $\infty$ controller design}

The representation of state space of a QoV model in the LPV framework by including an MR damper in the suspension considering straight direction and $\mathrm{D}=0$ can be defined as:

$$
\left[\begin{array}{c}
\dot{z}_{s} \\
\ddot{z}_{s} \\
\dot{z}_{u s} \\
\ddot{z}_{u s}
\end{array}\right]=A_{s}\left[\begin{array}{c}
z_{s} \\
\dot{z}_{s} \\
z_{u s} \\
\dot{z}_{u s}
\end{array}\right]+\left[\begin{array}{ll}
B_{s} & B_{s 1}
\end{array}\right]\left[\begin{array}{l}
u \\
z_{r}
\end{array}\right], y=C_{s}\left[\begin{array}{c}
z_{s} \\
\dot{z}_{s} \\
z_{u s} \\
\dot{z}_{u s}
\end{array}\right]
$$

where

$$
\begin{aligned}
& A_{S}=\left[\begin{array}{cccc}
0 & 1 & 0 & 0 \\
-\frac{k_{s}+k_{p}}{m_{s}} & -\frac{c_{p}}{m_{s}} & \frac{k_{s}+k_{p}}{m_{s}} & \frac{c_{p}}{m_{s}} \\
0 & 0 & 0 & 1 \\
\frac{k_{s}+k_{p}}{m_{u s}} & \frac{c_{p}}{m_{u s}} & \frac{-k_{s}-k_{p}-k_{t}}{m_{u s}} & -\frac{c_{p}}{m_{s}}
\end{array}\right]
\end{aligned}
$$

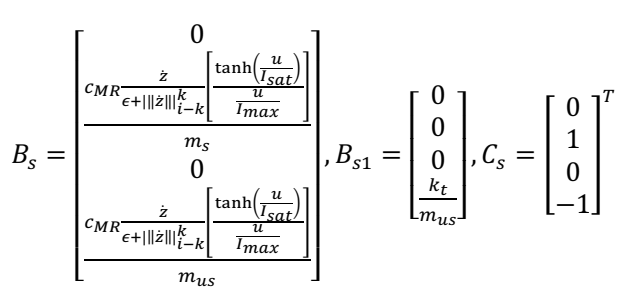

where $\mathrm{u}$ is the LPV QoV model exogenous input. The MR damper is represented by the Vmax model [5]:

$$
\begin{gathered}
F_{D}=c_{p} \dot{z}+k_{p} z+I c_{M R} \rho \\
\rho=\dot{z} \frac{1}{\|\dot{z}\|_{i-k}^{i}+\epsilon}
\end{gathered}
$$

where $\rho$ describes the damping force behavior due to the pre-yield and post-yield regimes of the MR fluid. In this case, a very narrow pre-yield zone is assumed in order to emphasize the independence of the MR effect of the velocity.

The equations (1-3) were obtained by inserting equations (4-5) in the space state representation of a QoV model that lacks a damper. Thus, the passive damping force component is inserted in the A matrix, and the semi-active component force is inserted in the B matrix. The electric current, input of the VMax model, is represented as variable $u$. The tanh function saturates the model input. According to the system, equation (1), the input constraints on the LPV QoV model are:

1) Semiactiveness. The input of the QoV LPV system, equation (1) must be positive, [7]:

$$
u \geq 0, \forall t \geq 0
$$

where $u$ is the electric current to be applied. In order to take into account this input constraint, the absolute value of the LPV-based controller filtered output, $u_{f}$, is defined as equal to the electric current magnitude to be applied, $u=\left|u_{f}\right|$.

2) Saturation. The control input provided by the semiactive damper must be bounded to the finite interval between $\left[0, I_{\max }\right]$ A. First, the MR damper model included in the LPV QoV model is:

$$
F_{D}=c_{p} \dot{Z}+k_{p} z+c_{M R} \frac{\dot{z}}{\epsilon+\mid\|\dot{z}\| \|_{i-k}^{k}}\left[\frac{\tanh \left(\frac{u}{I_{s a t}}\right)}{\frac{u}{I_{\max }}}\right] u
$$

Hence, the proposed solution to saturation can be seen by simplifying equation (7), deriving on:

$$
F_{D}=c_{p} \dot{z}+k_{p} z+c_{M R} I_{\text {max }} \tanh \left(\frac{u}{I_{s a t}}\right) \frac{\dot{z}}{\epsilon+\mid\|\dot{z}\| \|_{i-k}^{k}}
$$

equation (8) is a version of the $V_{\text {Max }}$ model where the electric current input is saturated to $I_{\max }$. The term $c_{M R} I_{\text {max }}$ defines the maximum $M R$ force $F_{M R}$ to be applied in the whole velocity span. The term:

$$
\frac{\dot{z}}{\epsilon+|\|\dot{z}\||_{i-k}^{k}}
$$

represents the sigmoid form of the $M R$ force component oscillating between -1 and 1 ; it assigns the sign to the force component and the maximum $M R$ force for the maximum velocity in the last $k$ samples. The term:

$$
\tanh \left(\frac{u}{I_{\text {sat }}}\right)
$$

oscillates between 0 and 1 , where $I_{\text {sat }}$ defines the saturation slope of the electric current to be applied, as well as limits the exogenous input, $u_{c}$. High values allow $I_{\text {sat }}$ a fast saturation, and low values get slow saturation. Therefore, this parameter limits the maximum electric current to be applied. Higher values of $I_{\text {sat }}$ will obtain a fast response of the change between $I_{\min }$ and $I_{\max }$ (i. e. a road holding oriented $L P V$ control), while lower values will obtain a slow response (i. e. a comfort oriented $L P V$ control). The value of $I_{s a t}$ is obtained from simulations of the frequency responses of the vertical acceleration and tire deflection to observe which values of $I_{\text {sat }}$ are better for comfort, road holding, or both.

According to equation (7), the measurable and scheduling parameter is defined. The parameter is:

$$
\rho^{*}=\frac{\dot{z}}{\epsilon+\mid\|\dot{z}\| \|_{i-k}^{k}}\left[\frac{\tanh \left(\frac{u}{I_{\text {Iat }}}\right)}{\frac{u}{I_{\text {max }}}}\right], \rho^{*} \in[-1,1]
$$

where $u$ represents the electric current magnitude which is proportional to the maximum damping force to be applied by the $M R$ damper and the piston velocity modifies the damping coefficient. Equation (7) derives on:

$$
F_{D}=c_{p} \dot{z}+k_{p} z+c_{M R} \rho^{*} u
$$


3) Dissipativity. The damping coefficient must always be positive. This is shown using the $M R$ force component, $F_{M R}$ :

$$
F_{M R}=c_{M R} u \frac{\dot{z}}{\epsilon+|\|\dot{z}\||_{i-k}^{k}}\left[\frac{\tanh \left(\frac{u}{I_{\text {sat }}}\right)}{\frac{u}{I_{\text {max }}}}\right]
$$

and dividing equation (13) by $z$ derivative, the variable damping coefficient $\left(c_{u}\right)$ is obtained:

$$
c_{u}=\frac{F_{M R}}{\dot{z}}=\frac{I_{\max } c_{M R}}{\epsilon+\mid\|\dot{z}\| \|_{i-k}^{k}} \tanh \left(\frac{u}{I_{s a t}}\right)
$$

where $c_{u}$ is always positive since $I_{\max }$ and $c_{M R}$ are constant, and the terms $\epsilon+\mid\|\dot{z}\|_{i-k}^{k}$ and $\tanh \left(\frac{u}{I_{s a t}}\right)$ are always positive.

4) Gain of the MR mechanism. The maximum damping coefficient generated by the $M R$ damper must be as close as possible to the critical damping coefficient of the mass of interest. If the $M R$ damping is not enough, a gain of damping force $G I$ is needed, allowing the $M R$ damper to equal the required critical damping coefficient of the sprung mass or the unsprung mass in order to achieve the control goals. A continuously variable semiactive suspension should have an off-state damping ratio of 0.1 to 0.2 and an on-state damping ratio of 1.0 or greater, [8]. These design values will improve ride comfort and suspension deflection while approximately maintaining the same road holding as the passive suspension. If the semi-active suspension control goals include the comfort and the road holding, the critical damping of the unsprung mass for an ideal damping ratio, $\xi=1.0$ must be equal to the maximum damping coefficient of the $M R$ damper:

$$
2 \sqrt{\left(k_{s}+k_{t}\right) m_{u s}} \xi=\frac{\overline{F_{D}(\dot{z})}}{\dot{z}}=G I I_{\max } c_{M R}+G I c_{p}
$$

where $\frac{\overline{F_{D}(\dot{z})}}{\dot{z}}$ is the maximum damping force that the $M R$ damper can dissipate. If the goal is only the comfort, the critical damping of the sprung mass for an ideal damping ratio, $\xi=1.0$, must be equal to the maximum damping coefficient of the $M R$ damper:

$$
2 \sqrt{k_{r} m_{s}} \xi=\frac{\overline{F_{D}(\dot{z})}}{\dot{z}}=G I I_{\max } c_{M R}+G I c_{p}
$$

where $k_{r}$ is the effective stiffness of the suspension and tire springs called ride rate, $k_{r}=\frac{\eta^{2} k_{s}+k_{t}}{\eta^{2} k_{s} k_{t}}$. The computed gain $G I$ will be included in the $Q o V$ model used in the $L P V$ controller synthesis. This gain ensures the controller output considers the critical damping ratio of the $Q o V$ model. The parameters $c_{p}$ and $c_{M R}$ in matrices $A_{s}$ and $B_{S}$ will be multiplied by the gain $G I$ :

$$
A^{*}{ }_{s}=\left[\begin{array}{cccc}
0 & 1 & 0 & 0 \\
-\frac{k_{s}+k_{p}}{m_{s}} & -\frac{G I c_{p}}{m_{s}} & \frac{k_{s}+k_{p}}{m_{s}} & \frac{G I c_{p}}{m_{s}} \\
0 & 0 & 0 & 1 \\
\frac{k_{s}+k_{p}}{m_{u s}} & \frac{G I c_{p}}{m_{u s}} & \frac{-k_{s}-k_{p}-k_{t}}{m_{u s}} & -\frac{G I c_{p}}{m_{s}}
\end{array}\right], B^{*}{ }_{s}=\left[\begin{array}{c}
0 \\
-\frac{G I c_{M R} \rho^{*}}{m_{s}} \\
0 \\
\frac{G I c_{M R} \rho^{*}}{m_{u s}}
\end{array}\right]
$$

\subsection{Controller synthesis}

The generalized system for the $\mathrm{H}_{x} / \mathrm{LPV}$ controller synthesis for one scheduling parameter is not proper for the $L P V$-based controller synthesis. According to the definition presented in [2] for an ideal linear design of a $L P V$ system for the controller synthesis, a proper filter, equation (18) is added to the input of the $L P V$ system, equation (1):

$$
F_{1}:\left(\begin{array}{l}
\dot{x}_{f} \\
u_{f}
\end{array}\right)=\left[\begin{array}{cc}
A_{f} & B_{f} \\
C_{f \rho} & 0
\end{array}\right]\left(\begin{array}{l}
x_{f} \\
u_{o}
\end{array}\right)
$$

In this way, $\rho^{*}$ will be in the states transition matrix $A^{*}{ }_{S}$. Fig. 1 shows the obtained $Q o V$ structure by using the $M R$ damper model with saturated input, equation (8) and the ideal linear design.

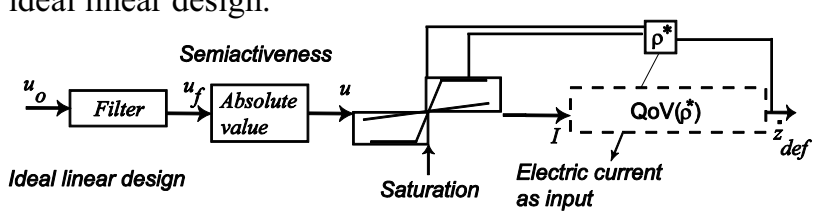

Figure 1. Model with a semi-active bounded input saturation.

Hence, the new proposed $L P V$ system, equation (19), to perform the $L P V$ controller synthesis is defined by the scheduling variable $\rho *$ :

$$
\left[\begin{array}{c}
\dot{z}_{s} \\
\ddot{z}_{s} \\
\dot{z}_{u s} \\
\ddot{z}_{u s} \\
\dot{x}_{f}
\end{array}\right]=A\left(\rho^{*}\right)\left[\begin{array}{c}
z_{s} \\
\dot{z}_{s} \\
z_{u s} \\
\dot{z}_{u s} \\
x_{f}
\end{array}\right]+B u+B_{1} w, y=C\left[\begin{array}{c}
z_{s} \\
\dot{z}_{s} \\
z_{u s} \\
\dot{z}_{u s} \\
\dot{x}_{f}
\end{array}\right]
$$

where

$$
\begin{gathered}
A\left(\rho^{*}\right)=\left[\begin{array}{cc}
A^{*} & \rho^{*} B^{*}{ }_{s} C_{f} \\
0 & A_{f}
\end{array}\right], B=\left(\begin{array}{c}
0 \\
B_{f}
\end{array}\right) \\
B_{1}=\left(\begin{array}{c}
B_{s 1} \\
0
\end{array}\right), \quad C=\left(\begin{array}{c}
C_{s} \\
0
\end{array}\right)^{T}
\end{gathered}
$$

and the electric current to be applied is a function of $\mathrm{u}$ :

$$
I(u)=I_{\text {max }} \tanh \left(\frac{u}{I_{\text {sat }}}\right)
$$

In order to meet the control specifications, two $H \infty$ weighting functions, $W_{\ddot{z}_{s}}$ and $W_{z_{u s}-z_{r}}$, obtained in case lin [9], were used according to the comfort performance without affecting the road holding, Fig. 2.

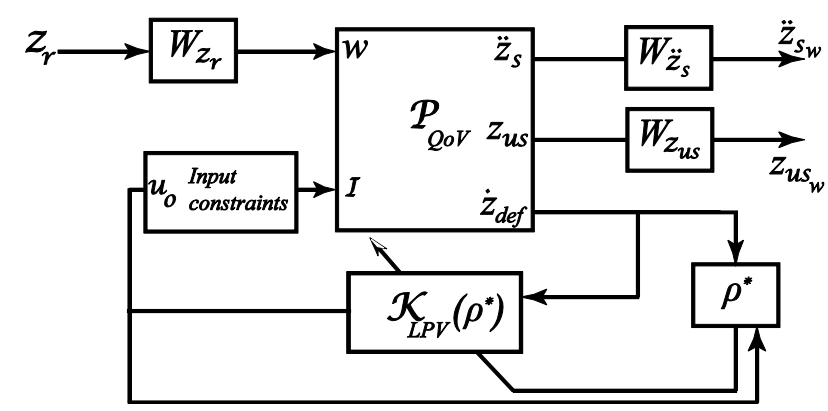

Figure 2. The LPV control approach for the QoV model with semiactive suspensión.

The $L P V$-based controller was obtained through the solution of a Linear Matrix Inequalities problem, [2]. The controlled output vector is:

$$
z=\left(\ddot{z}_{s}, z_{u s}\right)^{T}
$$


Three $L P V$-based controllers were synthesized according to:

$$
2 \sqrt{k_{s} m_{s}} \xi=7,945 \mathrm{~N} / \mathrm{m}
$$

that corresponds to a damping ratio $\xi=1.1$ in the resonant frequency of the unsprung mass of the $Q o V$ model. By solving equation (16), one gets the desired gain of the damping value GI which will allow to apply the appropriate value of electric current:

$$
G I=\frac{2 \sqrt{\left(k_{s}+k_{t}\right) m_{u s}} \xi}{I_{\max c_{M R}+c_{p}}}=\frac{7912}{3192}=2.72
$$

where the values of equation (25) were obtained from an experimental lumped QoV lumped parameter model for $\mathrm{k}_{\mathrm{s}}, \mathrm{k}_{\mathrm{t}}$, and $\mathrm{m}_{\mathrm{us}}$; as well as the values of $\mathrm{c}_{\mathrm{p}}$ and $\mathrm{c}_{\mathrm{MR}}$. A maximum electric current $\mathrm{I}_{\max }=5 \mathrm{~A}$ was defined according to the industrial practice for this type of MR damper. The values of $I_{\text {sat }}$ were $0.8,1.6$, and 2.8 for road holding, tradeoff and comfort oriented performances whose LPV-based controllers are named LPV-Road holding, LPV-tradeoff, and LPV-Comfort, respectively.

\subsection{Control schemes validation}

The nomenclature, validation and the set of the benchmark controllers are done and specified according to [5].

\section{Results and discussion}

The nonlinear QoV model was simulated with the controllers specified as specified in [10] using the test based on pseudobode test [2], Fig. 3.

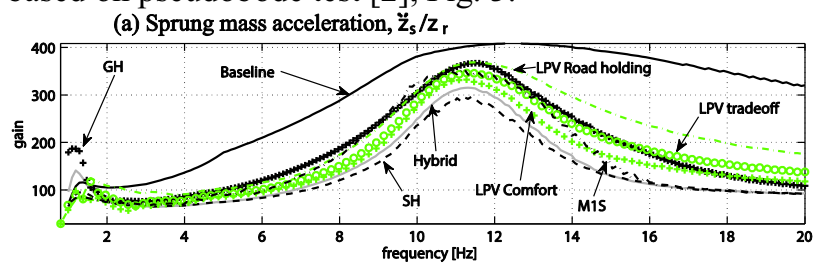

(b) Tire deflection, $\left(\mathrm{z}_{\mathrm{us}}-\mathrm{z}_{\mathrm{r}}\right) / \mathrm{z}_{\mathrm{r}}$

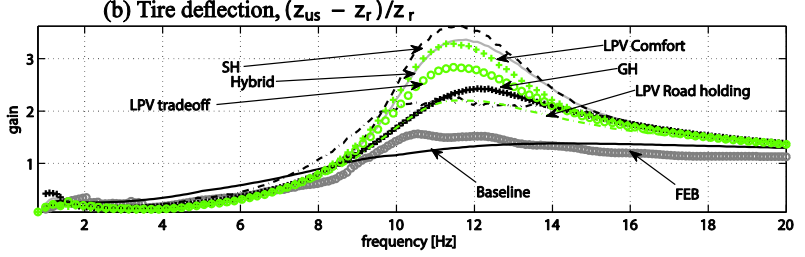

Figure 3. Pseudo-Bode for transfer functions in a closed loop simulation for a nonlinear QoV model: (a) sprung mass acceleration, (b) tire deflection.

The baseline suspension was also simulated. It must be noted that all the controllers are better than the baseline suspension in comfort, Fig. 3(a). The proposed controller (LPV-based) have a better performance below $1.6 \mathrm{~Hz}$ than the Hybrid controller. In the secondary ride frequencies, the LPV-Co, LPV-Tradeoff, Hybrid controllers offer good gains. Qualitatively, it can be said that the proposed approaches are better than the Hybrid controller in the primary ride, and they have similar performances in the tirehop resonant frequency. When dealing with the road holding goal, the best performance corresponds to the GH controller. The LPVComfort, $\mathrm{SH}$ and Hybrid controller are not well suited for this performance, see Figure 3c. The LPV Road holding controllers behave better than the $\mathrm{GH}$ controller. The Hybrid controller does not achieve a good tradeoff performance for comfort and road holding at the same time. Also, the baseline suspension is optimized for the suspension deflection and road holding with a sensitive payload in comfort. The frequency domain analysis validates the LPV-based proposed controllers as the best options in comfort and road holding performances.

The implementation of this LPV controller design based on one parameter case must be explored using a synthesis for LPV controllers with low implementation complexity, [10], in order to validate it using an experimental test rig and in an in-vehicle tests.

\section{Conclusion}

In summary, numerical results show $L P V$-based controllers as the best options to control comfort and road holding performances simultaneously. The jerk in the sprung mass in controllers as $\mathrm{SH}, \mathrm{GH}$ and Hybrid is present in numerical results, while the proposed controllers do not have this problem. If the GI parameter reacts to a road estimation then GI can be proposed as an adjustment of the semi-active suspension according to the road.

\section{References}

1. P. Barak, No. 922140, SAE Technical Paper, (1992)

2. C. Poussot-Vassal, O. Sename, L. Dugard, P. Gaspar, Z. Szabo, J. Bokor, CEP 16, 17 (2008)

3. S. M. Savaresi, C. Spelta, JDSMC 129, 11 (2007)

4. F. D. Goncalves, M. Ahmadian, SHOCK VIB 10, 11 (2003)

5. J. de-J. Lozoya-Santos, R. Morales-Menendez, R. A. Ramírez-Mendoza, MATH PROB ENG 2012, (2012)

6. J. Mohammadpour, C. W. Scherer, Springer Science \& Business Media, 2012

7. A. L. Do, O. Sename, L. Dugard, American Control Conference (ACC), 2010.

8. L. R. Miller, Proceedings of the 27th IEEE Conference on In Decision and Control, (1988)

9. A. L. Do, S. Boussaad, J de-J. Lozoya-Santos, O. Sename, L. Dugard, R. A. Ramirez-Mendoza, 12th Mini conference on vehicle system dynamics, identification and anomalies (VSDIA) (2010)

10. C. Hoffmann, S. M. Hashemi, H. S. Abbas and H. Werner, IEEE T CONTR SYST T, 22, 6 (2014) 